Bull. Korean Math. Soc. 48 (2011), No. 2, pp. 303-314

DOI 10.4134/BKMS.2011.48.2.303

\title{
PRECONDITIONED GAUSS-SEIDEL ITERATIVE METHOD FOR $Z$-MATRICES LINEAR SYSTEMS
}

\author{
Hailong Shen, Xinhui Shao, Zhenxing Huang, and Chunji Li
}

\begin{abstract}
For $A x=b$, it has recently been reported that the convergence of the preconditioned Gauss-Seidel iterative method which uses a matrix of the type $P=I+S(\alpha)$ to perform certain elementary row operations on is faster than the basic Gauss-Seidel method. In this paper, we discuss the adaptive Gauss-Seidel iterative method which uses $P=I+S(\alpha)+\bar{K}(\beta)$ as a preconditioner. We present some comparison theorems, which show the rate of convergence of the new method is faster than the basic method and the method in [7] theoretically. Numerical examples show the effectiveness of our algorithm.
\end{abstract}

\section{Introduction}

We consider iterative methods for solving a linear system

$$
A x=b,
$$

where $A$ is an $n \times n$ matrix with unit diagonal elements, $x$ and $b$ are $n$ dimensional vectors.

If we write $A=M-N$ with a nonsingular matrix $M$, then the basic iterative scheme for (1) is defined by

$$
M x_{k+1}=N x_{k}+b, \quad k=0,1,2, \ldots
$$

Let $T=M^{-1} N$ and $c=M^{-1} b$. Then (2) can be also be written as

$$
x_{k+1}=T x_{k}+c, \quad k=0,1,2, \ldots
$$

Writing $A=I-L-U$ and taking $M=I-L$ in (2) or (3) yield the classic Gauss-Seidel method, where $I$ is the identity matrix, and $L$ and $D$ are strictly lower and strictly upper triangular matrices, respectively. The matrix $T=$ $(I-L)^{-1} U$ is then called the Gauss-Seidel iteration matrix.

Received June 26, 2009; Revised September 10, 2009

2010 Mathematics Subject Classification. 65F08.

Key words and phrases. Gauss-Seidel iterative method, preconditioned method, $Z$ matrix, diagonal dominant matrix.

This project is supported by the National Natural Science Foundation of China (No. 11071033) and the Fundamental Research Funds for the Central Universities (No. 090405013). 
We now transform the original system (1) into the preconditioned form

$$
P A x=P b \text {. }
$$

Then the corresponding basic iterative scheme is

$$
M_{p} x_{k+1}=N_{p} x_{k}+P b, \quad k=0,1,2, \ldots,
$$

where $P A=M_{p}-N_{p}$ is a regular splitting of $P A$, and $M_{p}$ is a nonsingular matrix, the nonsingular matrix $P$ is called a preconditioner, the iterative method used in linear equations $P A x=P b$ is called the preconditioned iterative method.

In 1987, Milaszewiez [8] used the following preconditioner

$$
P=I+C=\left(\begin{array}{cccc}
1 & 0 & \cdots & 0 \\
-a_{21} & 1 & \cdots & 0 \\
\vdots & \vdots & \ddots & \vdots \\
-a_{n 1} & 0 & \cdots & 1
\end{array}\right)
$$

In 2003, Hadjidimos et al. [3] presented the following preconditioner

$$
P(\alpha)=I+C(\alpha)=\left(\begin{array}{ccccc}
1 & 0 & 0 & \cdots & 0 \\
-\alpha_{2} a_{21} & 1 & 0 & \cdots & 0 \\
-\alpha_{3} a_{31} & 0 & 1 & \cdots & 0 \\
\vdots & \vdots & \vdots & \ddots & \vdots \\
-\alpha_{n} a_{n 1} & 0 & 0 & \cdots & 1
\end{array}\right)
$$

The preconditioner $P(\alpha)$ in (7) is the general preconditioner of the matrix $P$ in (6), when $\alpha_{i}=1, i=2,3, \ldots$, the preconditioner became the matrix $P$.

In 1991, Gunawardena et al. [2] employed the following preconditioner

$$
P_{S}=I+S=\left(\begin{array}{ccccc}
1 & -a_{12} & 0 & \cdots & 0 \\
0 & 1 & -a_{23} & \cdots & 0 \\
0 & 0 & 1 & \ddots & \vdots \\
\vdots & \vdots & \vdots & \ddots & -a_{n-1, n} \\
0 & 0 & 0 & \cdots & 1
\end{array}\right)
$$

In 1997, Kohno et al. [5] employed the general preconditioned form of the matrix

(9) $\quad P_{S}(\alpha)=I+S(\alpha)=\left(\begin{array}{ccccc}1 & -\alpha_{1} a_{12} & 0 & \cdots & 0 \\ 0 & 1 & -\alpha_{2} a_{23} & \cdots & 0 \\ 0 & 0 & 1 & \ddots & \vdots \\ \vdots & \vdots & \vdots & \ddots & -\alpha_{n-1} a_{n-1, n} \\ 0 & 0 & 0 & \cdots & 1\end{array}\right)$.

When $\alpha_{i}=1, i=1,2, \ldots, n-1$, the preconditioner $P_{S}(\alpha)$ is equal to $P_{S}$. 
In [6], $P=I+S(\alpha)+K(\beta)$ is presented, where

$$
K(\beta)=\left\{\begin{array}{cc}
-\beta_{i} a_{i j}, & i=j+1, \\
0, & \text { otherwise }
\end{array}\right.
$$

The comparison theorem and numerical examples show that the method is the improvement for the iterative method in [5].

In this paper, we first consider the new preconditioner $P=I+S(\alpha)+\tilde{K}(\beta)$, where

$$
\tilde{K}(\beta)=\left\{\begin{array}{cc}
-\beta_{i} a_{i 1}, & i=2,3, \ldots, n, \\
0, & \text { otherwise. }
\end{array}\right.
$$

Next we discuss its convergence. Finally, we show with numerical examples that this method yields a considerable improvement in the rate of convergence for the iterative method.

\section{Basic results}

First, we need the following definitions and results.

Definition 1 ([4]). An $n \times n$ real matrix $A=\left(a_{i j}\right)$ is called $Z$-matrix, if $a_{i j} \leq 0, \forall i \neq j$, and $a_{i i}>0$; and an $A$ is called diagonal dominant (or strictly diagonal dominant) $Z$-matrix, if $\sum_{j=1}^{n} a_{i j} \geq 0$ (or $\sum_{j=1}^{n} a_{i j}>0$ ).

Definition 2 ([4]). A matrix $A$ is irreducible if there exists a permutation matrix $P$ such that

$$
P A P^{T}=\left(\begin{array}{cc}
A_{1} & 0 \\
A_{2} & A_{3}
\end{array}\right)
$$

where $A_{1}$ and $A_{3}$ are square matrices.

Definition $3([1])$. Let $A=\left(a_{i j}\right)_{n \times n}, B=\left(b_{i j}\right)_{n \times n}$. We say $A>B$ if $a_{i j}>$ $b_{i j}, \forall 1 \leq i, j \leq n$.

Definition $4([1])$. Let $A=\left(a_{i j}\right)_{n \times n}, B=\left(b_{i j}\right)_{n \times n}$. We say $A \geq B$ if $a_{i j} \geq$ $b_{i j}, \forall 1 \leq i, j \leq n$.

Lemma 1 ([4]). Let $A$ be a nonnegative matrix. Then

(1) A has a positive real eigenvalue equal to its spectral radius.

(2) To the spectral radius $\rho(A)$ there corresponds an eigenvector $x>0$.

(3) If $A$ is an irreducible matrix, then $\rho(A)>0$ and there exists $x>0$ such that $A x=\rho(A) x$.

Lemma 2 ([4]). Let $A$ be a nonnegative matrix. Then

(1) If $\alpha x \leq A x$ for some nonnegative vector $x \neq 0$, then $\alpha \leq \rho(A)$.

(2) $A$ is a irreducible matrix if and only if $(I+A)^{n-1}>0$.

(3) If $A x \leq \beta x$ for some positive vector $x$, then $\rho(A) \leq \beta$. Moreover, if $A$ is irreducible and if $0 \neq \alpha x \leq A x \leq \beta x, \alpha x \neq A x$ and $A x \neq \beta x$ for some nonnegative vector $x$, then $\alpha<\rho(A)<\beta$, and $x$ is a positive vector.

(4) If $B$ is an $n \times n$ matrix, $0 \leq A \leq B, A \neq B, A+B$ are all irreducible matrices, then $\rho(A)<\rho(B)$. 
Lemma $3([6])$. Let $A=D-E-F$ be a $Z$-matrix, where $D,-E$ and $-F$ are diagonal, strictly lower triangular and strictly upper triangular parts of $A$, respectively. Then an upper bound of the spectral radius for the Gauss-Seidel iterative matrix $\tilde{T}$ is given by

$$
\rho(T) \leq \max \frac{\tilde{f}_{i}}{\tilde{d}_{i}-\tilde{e}_{i}}, \quad \forall i,
$$

where $\tilde{d}_{i}, \tilde{e}_{i}$ and $\tilde{f}_{i}$ are sums of elements in the $i$-th row of $D, E$ and $F$, respectively.

\section{New preconditioned iterative method and convergence analysis}

We propose a preconditioned iterative method with

$$
P=I+S_{\alpha}^{\beta}=\left(\begin{array}{ccccc}
1 & -\alpha_{1} a_{12} & & & \\
-\beta_{2} a_{21} & 1 & -\alpha_{2} a_{23} & & \\
-\beta_{2} a_{21} & & 1 & \ddots & \\
\vdots & & & \ddots & -\alpha_{n-1} a_{n-1, n} \\
-\beta_{n} a_{n 1} & & & & 1
\end{array}\right),
$$

where $S_{\alpha}^{\beta}=S(\alpha)+\tilde{K}(\beta)$,

$$
\begin{aligned}
& S(\alpha)=\left(\begin{array}{ccccc}
0 & -\alpha_{1} a_{12} & 0 & \cdots & 0 \\
0 & 0 & -\alpha_{2} a_{23} & \ddots & \vdots \\
0 & 0 & 0 & \ddots & 0 \\
\vdots & \vdots & \vdots & \ddots & -\alpha_{n-1} a_{n-1, n} \\
0 & 0 & 0 & \cdots & 0
\end{array}\right), \\
& \tilde{K}(\beta)=\left(\begin{array}{ccccc}
0 & 0 & 0 & \cdots & 0 \\
-\beta_{2} a_{21} & 0 & 0 & \ddots & \vdots \\
-\beta_{3} a_{31} & 0 & 0 & \ddots & 0 \\
\vdots & \vdots & \vdots & \ddots & 0 \\
-\beta_{n} a_{n, 1} & 0 & 0 & \cdots & 0
\end{array}\right)
\end{aligned}
$$

Then

$$
\begin{aligned}
A_{\alpha}^{\beta} & =\left(I+S_{\alpha}^{\beta}\right) A=I-L-U+S_{\alpha}^{\beta}-S_{\alpha}^{\beta}(L+U), \\
b_{\alpha}^{\beta} & =\left(I+S_{\alpha}^{\beta}\right) b .
\end{aligned}
$$

Let $S_{\alpha}^{\beta}(L+U)=D_{0}+L_{0}+U_{0}$, where $D_{0}, L_{0}$ and $U_{0}$ are diagonal, strictly lower triangular and strictly upper triangular parts of $S_{\alpha}^{\beta}(L+U)$, respectively, and

$$
D_{0}=\operatorname{diag}\left\{\beta_{1} a_{12} a_{21}, \alpha_{2} a_{21} a_{12}+\beta_{2} a_{23} a_{32}, \ldots, \alpha_{n-1} a_{n-1,1} a_{1, n-1}\right.
$$




$$
\left.+\beta_{n-1} a_{n-1,1} a_{1, n-1}, \alpha_{n} a_{n, 1} a_{1, n}\right\},
$$

then

$$
A_{\alpha}^{\beta}=\left(I-D_{0}-L+S_{\alpha}-L_{0}\right)-\left(U-S^{\beta}+U_{0}\right)
$$

If

$$
\begin{cases}\alpha_{1} a_{12} a_{21} \neq 1, & i=1, \\ \alpha_{i} a_{i, i+1} a_{i+1, i}+\beta_{i} a_{i, 1} a_{1, i} \neq 1, & i=2, \ldots, n-1, \\ \beta_{n} a_{n, 1} a_{1, n} \neq 1, & i=n,\end{cases}
$$

then matrix $\left(I-D_{0}-L+S_{\alpha}-L_{0}\right)^{-1}$ exists and the Gauss-Seidel iteration matrix $T_{\alpha}^{\beta}$ for $A_{\alpha}^{\beta}$ is defined by

$$
T_{\alpha}^{\beta}=\left(I-D_{0}-L+S_{\alpha}-L_{0}\right)^{-1}\left(U-S^{\beta}+U_{0}\right) .
$$

Let

$$
\alpha=\operatorname{diag}\left(\alpha_{1}, \alpha_{2}, \ldots, \alpha_{n}\right), \quad \beta=\operatorname{diag}\left(\beta_{1}, \beta_{2}, \ldots, \beta_{n}\right)
$$

and

$$
S=\left(\begin{array}{cccc}
0 & -a_{12} & \cdots & 0 \\
0 & 0 & \ddots & \vdots \\
\vdots & \vdots & \ddots & -a_{n-1, n} \\
0 & 0 & \cdots & 0
\end{array}\right), \quad \tilde{K}=\left(\begin{array}{cccc}
0 & 0 & \cdots & 0 \\
-a_{21} & 0 & \ddots & \vdots \\
\vdots & \vdots & \ddots & 0 \\
-a_{n, 1} & 0 & \cdots & 0
\end{array}\right)
$$

Then $S_{\alpha}^{\beta}=S(\alpha)+\tilde{K}(\beta)=\alpha S+\beta \tilde{K}$. If $\alpha$ and $\beta$ are identity matrices, then

$$
\tilde{A}=(I+S+\tilde{K}) A=\tilde{D}-\tilde{L}-\tilde{U}, \quad \tilde{T}=(\tilde{D}-\tilde{L})^{-1} \tilde{U} .
$$

Obviously, when $\alpha_{i}=0, i=1,2, \ldots, n$, the new method in this paper becomes the method in [3]; when $\beta_{i}=0, i=1,2, \ldots, n$, the new method in this paper becomes the method in [5].

Theorem 1. Let $A \in R^{n \times n}(n \geq 3)$ be a nonsingular diagonal dominant $Z$ matrix with unit diagonal elements. If for $0<\alpha_{i} \leq 1,0<\beta_{i} \leq 1(i=1,2, \ldots, n)$, $A$ satisfies the following conditions, then $A_{\alpha}^{\beta}$ is a strictly diagonal dominant $Z$ matrix and $\rho\left(T_{\alpha}^{\beta}\right)<1$.

(1) $a_{i i}-\left|a_{i 1}\right|-\left|a_{i, i+1}\right|>0, a_{11}-\left|a_{12}\right|>0, a_{n n}-\left|a_{n 1}\right|>0$ for any $i=$ $2,3, \ldots, n-1$.

(2) If $\sum_{j=1}^{n} a_{i j}=0$ for $i=1,2, \ldots, n-1$, then $a_{i, 1} a_{i, i+1} \neq 0$.

(3) There exists at least a row for $i$-th row and $(i+1)$-th row such that it is strictly diagonal dominant row for any $i=1,2, \ldots, n-1$.

(4) There exists at least a strictly diagonal dominant row for the first row and $n$-th row; and if $\sum_{j=1}^{n} a_{n j}=0$, then $a_{n 1} \neq 0$.

Proof. In fact,

$$
\left(A_{\alpha}^{\beta}\right)_{i i}=a_{i i}-\alpha_{i} a_{i, i+1} a_{i+1, i}-\beta_{i} a_{i 1} a_{1 i} \geq a_{i i}-\left|a_{i, i+1}\right|-\left|a_{i, 1}\right|>0 .
$$


For $i>j, i=2, \ldots, n$, when $j=1$,

(15) $\left(A_{\alpha}^{\beta}\right)_{i 1}=a_{i 1}-\alpha_{i} a_{i, i+1} a_{i+1, i}-\beta_{i} a_{i 1} a_{11}=\left(1-\beta_{i}\right) a_{i 1}-\alpha_{i} a_{i, i+1} a_{i+1, i} \leq 0$, when $j=2, \ldots, n-1$,

$$
\left(A_{\alpha}^{\beta}\right)_{i j}=a_{i j}-\alpha_{i} a_{i, i+1} a_{i+1, j}-\beta_{i} a_{i 1} a_{1 j} \leq 0 .
$$

For $i<j, i=1,2, \ldots, n-1, j=2, \ldots, n$, when $i+1=j$,

$$
\left(A_{\alpha}^{\beta}\right)_{i, i+1}=\left(1-\alpha_{i}\right) a_{i, i+1}-\beta_{i} a_{i 1} a_{1, i+1} \leq 0 .
$$

Otherwise

$$
\left(A_{\alpha}^{\beta}\right)_{i j}=a_{i, j}-\alpha_{i} a_{i, i+1} a_{i+1, j}-\beta_{i} a_{i 1} a_{1 j} \leq 0 .
$$

Hence, $A_{\alpha}^{\beta}$ is a $Z$-matrix.

From (2), (3) and (4), there exists at least an inequality such that it holds for the following inequalities:

$$
\sum_{j=1}^{n} a_{i j}>0, \quad-\alpha_{i} a_{i, i+1} \sum_{j=1}^{n} a_{i+1, j}>0, \quad-\beta_{i} a_{i 1} \sum_{j=1}^{n} a_{1 j}>0 .
$$

Then

$$
\sum_{j=1}^{n}\left(A_{\alpha}^{\beta}\right)_{i j}=\sum_{j=1}^{n} a_{i j}-\alpha_{i} a_{i, i+1} \sum_{j=1}^{n} a_{i+1, j}-\beta_{i} a_{i 1} \sum_{j=1}^{n} a_{1 j}>0 .
$$

Hence, we know that $A_{\alpha}^{\beta}$ is a strictly diagonal dominant $Z$-matrix.

Now we decompose $A_{\alpha}^{\beta}$, let $A_{\alpha}^{\beta}=D_{\alpha}^{\beta}-L_{\alpha}^{\beta}-U_{\alpha}^{\beta}$, where $D_{\alpha}^{\beta},-L_{\alpha}^{\beta}$ and $-U_{\alpha}^{\beta}$ are diagonal, strictly lower triangular and strictly upper triangular parts of $A_{\alpha}^{\beta}$, respectively, and let $d_{\alpha, i}^{\beta}, l_{\alpha, i}^{\beta}$ and $u_{\alpha, i}^{\beta}$ are the sum of $i$-th row elements of $D_{\alpha}^{\beta}$, $L_{\alpha}^{\beta}$ and $U_{\alpha}^{\beta}$, respectively. From (18), we have $d_{\alpha, i}^{\beta}-l_{\alpha, i}^{\beta}-u_{\alpha, i}^{\beta}>0$; and from (17) and (18), we have $u_{\alpha, i}^{\beta} \geq 0$. So

$$
\frac{u_{\alpha, i}^{\beta}}{d_{\alpha, i}^{\beta}-l_{\alpha, i}^{\beta}}<1
$$

From Lemma 3, $\rho\left(T_{\alpha}^{\beta}\right)<1$. The proof is complete.

Theorem 2. Let $A=I-L-U$ be a $Z$-matrix, $0<a_{i 1} a_{1 i}+a_{i, i+1} a_{i+1, i}<1$ for $i=2, \ldots, n-1$, and $0<a_{12} a_{21}<1,0<a_{n 1} a_{1 n}<1$. If $\rho(T)=1$, then $\rho(\tilde{T})=\rho(T)=1$.

Proof. It is obviously that $\tilde{A}$ in (14) is a $Z$-matrix. Since

$$
\begin{aligned}
\tilde{A} & =(I+\tilde{K}+S) A=(I+\tilde{K}+S)(I-L-U) \\
& =I+\tilde{K}-L-S L-\tilde{K} L-\tilde{K} U-U-S U+S,
\end{aligned}
$$


we have $-\tilde{K} L=0, I+\tilde{K}-L-S L$ is a lower triangular matrix; $-U-S U+S$ is an upper triangular matrix. But

$$
-\tilde{K} U=\left(\begin{array}{ccccc}
0 & 0 & 0 & \cdots & 0 \\
0 & -a_{21} a_{12} & -a_{21} a_{13} & \cdots & -a_{21} a_{1 n} \\
0 & -a_{31} a_{12} & -a_{31} a_{13} & \cdots & -a_{31} a_{1 n} \\
\vdots & \vdots & \vdots & & \vdots \\
0 & -a_{n 1} a_{12} & -a_{n 1} a_{13} & \cdots & -a_{n 1} a_{1 n}
\end{array}\right) .
$$

If $-\tilde{K} U=M_{1}+M_{2}$, where $M_{2}$ is a strictly upper triangular part of $-\tilde{K} U$, then

$$
\tilde{T}=(\tilde{D}-\tilde{L})^{-1} \tilde{U}=\left(I+\tilde{K}-L-S L+M_{1}\right)^{-1}\left(U+S U-S-M_{2}\right) .
$$

Hence $T$ and $\tilde{T}$ are nonnegative matrices, and

$$
T=\left(\begin{array}{cc}
0 & T_{12} \\
0 & T_{22}
\end{array}\right), \quad \tilde{T}=\left(\begin{array}{cc}
0 & \tilde{T}_{12} \\
0 & \tilde{T}_{22}
\end{array}\right),
$$

where $T_{22}$ and $\tilde{T}_{22}$ are all $(n-1) \times(n-1)$ irreducible matrices. From $\rho(T)=1$, we have $\rho\left(T_{22}\right)=1$.

From Lemma $4, T_{22} x^{\prime}=x^{\prime}$ for some vector $x^{\prime}>0$. Now we construct the positive vector $x=\left(\begin{array}{c}T_{12} x^{\prime} \\ x^{\prime}\end{array}\right)$, it satisfies $T x=x$.

Since $(I-L)^{-1} U x=x, U x=(I-L) x$ and

$$
\tilde{T} x=\left(I+\tilde{K}-L-S L+M_{1}\right)^{-1}\left(U+S U-S-M_{2}\right) x,
$$

so we have

$$
\begin{aligned}
\left(U+S U-S-M_{2}\right) x & =\left(U+S U-S+\tilde{K} U+M_{1}\right) x \\
& =U x+S U x-S x+\tilde{K} U x+M_{1} x \\
& =(I-L) x+S(I-L) x-S x+\tilde{K}(I-L) x+M_{1} x \\
& =\left(I+\tilde{K}-L-S L+M_{1}\right) x .
\end{aligned}
$$

Hence $\tilde{T} x=x$. From Lemma 2, we have $\rho(\tilde{T})=1$. The proof is complete.

Theorem 3. Let $A=I-L-U$ be an $n \times n(n \geq 3) Z$-matrix. $0<a_{i 1} a_{1 i}+$ $a_{i, i+1} a_{i+1, i}<1$ for $i=2, \ldots, n-1$, and $0<a_{12} a_{21}<1,0<a_{n 1} a_{1 n}<1$. Then

(1) $\rho(\tilde{T})<\rho(T)$ for $\rho(T)<1$,

(2) $\rho(\tilde{T})>\rho(T)$ for $\rho(T)>1$.

Proof. From Theorem 2, there exists a positive vector $x=\left(\begin{array}{c}T_{12} x^{\prime} \\ \rho(T) x^{\prime}\end{array}\right)$ such that $T x=\rho(T) x$. We have

$$
\tilde{T} x-T x
$$




$$
\begin{aligned}
& =(\tilde{D}-\tilde{L})^{-1}\left(U+S U-S-M_{2}\right) x-(I-L)^{-1} U x \\
& =(\tilde{D}-\tilde{L})^{-1}\left[\left(U+S U-S-M_{2}\right) x-(\tilde{D}-\tilde{L}) \rho(T) x\right] \\
& =(\tilde{D}-\tilde{L})^{-1}\left[\left(U+S U-S-M_{2}\right) x-\left(I+\tilde{K}-L-S L+M_{1}\right) \rho(T) x\right] \\
& =(\tilde{D}-\tilde{L})^{-1}\left[\rho(T) S x-S x+M_{1} x-\rho(T) M_{1} x\right] \\
& =(\rho(T)-1)(\tilde{D}-\tilde{L})^{-1}\left(S-M_{1}\right) x .
\end{aligned}
$$

We know easily that $(\tilde{D}-\tilde{L})^{-1}\left(S-M_{1}\right)$ is a nonnegative matrix, so $\tilde{T} x \leq$ $T x=\rho(T) x$ when $\rho(T)<1 ; \tilde{T} x \geq T x=\rho(T) x$ when $\rho(T)>1$. From (20), Lemma 4 and Lemma $5, \rho(\tilde{T})<\rho(T)$ for $\rho(T)<1$, and $\rho(\tilde{T})>\rho(T)$ for $\rho(T)>1$. The proof is complete.

Theorem 4. Let $A=I-L-U$ be a $Z$-matrix. $0<a_{i 1} a_{1 i}+a_{i, i+1} a_{i+1, i}<1$ for $i=2, \ldots, n-1$, and $0<a_{12} a_{21}<1,0<a_{n 1} a_{1 n}<1$. Then for any $0 \leq \alpha_{i} \leq 1,0 \leq \beta_{i} \leq 1$, we have

(1) $\rho\left(T_{\alpha}^{\beta}\right)<\rho(T)$ for $\rho(T)<1$,

(2) $\rho\left(T_{\alpha}^{\beta}\right)>\rho(T)$ for $\rho(T)>1$.

Proof. Since

$$
\begin{aligned}
T_{\alpha}^{\beta} x-T x & =\left(D_{\alpha}^{\beta}-L_{\alpha}^{\beta}\right)^{-1}\left(U+\alpha S U-\alpha S-\beta M_{2}\right) x-(I-L)^{-1} U x \\
& =(\rho(T)-1)\left(D_{\alpha}^{\beta}-L_{\alpha}^{\beta}\right)^{-1}\left(S-\beta M_{1}\right) x,
\end{aligned}
$$

and $0 \leq \alpha_{i} \leq 1,0 \leq \beta_{i} \leq 1,\left(D_{\alpha}^{\beta}-L_{\alpha}^{\beta}\right)^{-1}\left(S-\beta M_{1}\right)$ is also a nonnegative matrix. Then we have $T_{\alpha}^{\beta} x \leq T x=\rho(T) x$ for $\rho(T)<1 ; T_{\alpha}^{\beta} x \geq T x=\rho(T) x$ for $\rho(T)>1$. Hence we have our conclusion.

\section{Numerical examples}

Example 1. Let

$$
A=\left(\begin{array}{ccc}
1 & -0.4 & -0.3 \\
-0.5 & 1 & -0.2 \\
-0.6 & -0.5 & 1
\end{array}\right), \quad b=\left(\begin{array}{c}
0.3 \\
0.3 \\
-0.1
\end{array}\right)
$$

It is obviously that $A$ is a nonsingular $Z$-matrix, but it is not diagonal dominant matrix, so it does not satisfy the conditions in [5]. At the same time,

$$
(I+S) A=\left(\begin{array}{ccc}
0.8 & 0 & -0.38 \\
-0.62 & 0.9 & 0 \\
-0.6 & -0.5 & 1
\end{array}\right)
$$


is not strictly diagonal dominant $Z$-matrix. But

$$
\tilde{A}=(I+S+\tilde{K}) A=\left(\begin{array}{ccc}
0.8 & 0 & -0.38 \\
-0.12 & 0.7 & -0.15 \\
0 & -0.74 & 0.82
\end{array}\right)
$$

is a strictly diagonal dominant $Z$-matrix.

Now the classical Gauss-Seidel iterative method and the preconditioned Gauss-Seidel iterative method are considered for solving the linear system of equation (1). The stopping criterion $\left\|r_{k}\right\|_{\infty}<10^{-3}$ was used in the computations, where $r_{k}=b-A x^{(k)}$ and $x^{(k)}$ is the $k$-th iteration for each of the methods. The number of iterations (IT) for convergence needed for the classical Gauss-Seidel iterative method and the preconditioned Gauss-Seidel iterative method are listed in Table 1 and Table 2, respectively. Thus it can be seen that the preconditioned Gauss-Seidel method proposed in this paper has a faster convergence rate than that of the classical Gauss-Seidel iterative method. Let $x^{(0)}=(0,0,0)^{T}$, the classical Gauss-Seidel iterative method took 16 iterations for the convergence, while it took 6 iterations for the convergence of the preconditioned Gauss-Seidel method. But the two methods took the same CPU times.

Table 1. Numerical results of classical Gauss-Seidel method

\begin{tabular}{|c|c|c|c|c|}
\hline Ite. & $x_{1}^{(k)}$ & $x_{2}^{(k)}$ & $x_{3}^{(k)}$ & $\left\|x^{(k)}-x^{*}\right\|_{\infty}$ \\
\hline 0 & 0 & 0 & 0 & 1 \\
\hline 1 & 0.3000 & 0.4500 & 0.3050 & 0.7000 \\
\hline 2 & 0.5215 & 0.6467 & 0.5663 & 0.4337 \\
\hline 3 & 0.7286 & 0.7775 & 0.7259 & 0.2741 \\
\hline 4 & 0.8288 & 0.8596 & 0.8271 & 0.1729 \\
\hline 5 & 0.8920 & 0.9114 & 0.8909 & 0.1091 \\
\hline 6 & 0.9318 & 0.9441 & 0.9311 & 0.0689 \\
\hline 7 & 0.9570 & 0.9647 & 0.9565 & 0.0435 \\
\hline 8 & 0.9728 & 0.9777 & 0.9726 & 0.0274 \\
\hline 9 & 0.9829 & 0.9859 & 0.9827 & 0.0173 \\
\hline 10 & 0.9892 & 0.9911 & 0.9891 & 0.0109 \\
\hline 11 & 0.9932 & 0.9944 & 0.9931 & 0.0069 \\
\hline 12 & 0.9957 & 0.9965 & 0.9957 & 0.0044 \\
\hline 13 & 0.9973 & 0.9978 & 0.9973 & 0.0027 \\
\hline 14 & 0.9983 & 0.9986 & 0.9983 & 0.0017 \\
\hline 15 & 0.9989 & 0.9991 & 0.9989 & 0.0011 \\
\hline 16 & 0.9993 & 0.9994 & 0.9993 & 0.0007 \\
\hline
\end{tabular}


Table 2. Numerical results of preconditioned Gauss-Seidel method with $P=I+S+\tilde{K}$.

\begin{tabular}{|c|c|c|c|c|}
\hline Ite. & $x_{1}^{(k)}$ & $x_{2}^{(k)}$ & $x_{3}^{(k)}$ & $\left\|x^{(k)}-x^{*}\right\|_{\infty}$ \\
\hline 0 & 0 & 0 & 0 & 1 \\
\hline 1 & 0.5250 & 0.7043 & 0.7331 & 0.4750 \\
\hline 2 & 0.8732 & 0.9211 & 0.9288 & 0.1268 \\
\hline 3 & 0.9662 & 0.9789 & 0.9810 & 0.0338 \\
\hline 4 & 0.9910 & 0.9944 & 0.9949 & 0.0090 \\
\hline 5 & 0.9976 & 0.9985 & 0.9986 & 0.0024 \\
\hline 6 & 0.9994 & 0.9996 & 0.9996 & 0.0006 \\
\hline 7 & 0.9998 & 0.9999 & 0.9999 & 0.0002 \\
\hline 8 & 1.0000 & 1.0000 & 1.0000 & 0.0000 \\
\hline
\end{tabular}

Example 2. Let

$$
A=\left(\begin{array}{ccccc}
1 & -0.1 & -0.2 & -0.01 & 0 \\
-0.4 & 1 & -0.4 & 0 & -0.1 \\
-0.2 & -0.5 & 1 & -0.1 & -0.1 \\
-0.45 & -0.1 & -0.2 & 1 & -0.1 \\
-0.1 & -0.12 & -0.2 & -0.6 & 1
\end{array}\right)
$$

Then we report the spectral radius of three iterative methods in Table 3 .

Table 3. Comparisons of the three iterative methods in [5], [7] and this paper

\begin{tabular}{|l|l|l|l|l|l|l|}
\hline$\left(\alpha_{i}, \beta_{i}\right)$ & $(0.7,0.7)$ & $(0.9,0.9)$ & $(0.8,1)$ & $(1,1)$ & $(0.8,0.9)$ & $(0.9,1)$ \\
\hline GS1: $\rho(I+\alpha S)$ & 0.4059 & 0.3629 & 0.3848 & 0.3403 & 0.3848 & 0.3629 \\
\hline GS2: $\rho(I+\alpha S+\beta K)$ & 0.3785 & 0.3442 & 0.3540 & 0.3309 & 0.3572 & 0.3422 \\
\hline GS3: $\rho(I+\alpha S+\beta \tilde{K})$ & 0.3590 & 0.2947 & 0.2385 & 0.2651 & 0.3196 & 0.2851 \\
\hline
\end{tabular}

The above matrix $S$ is the form in [5], matrices $K$ and $\tilde{K}$ are as follows:

$$
K=\left(\begin{array}{ccccc}
0 & 0 & 0 & 0 & 0 \\
0.4 & 0 & 0 & 0 & 0 \\
0 & 0.5 & 0 & 0 & 0 \\
0 & 0 & 0.2 & 0 & 0 \\
0 & 0 & 0 & 0.6 & 0
\end{array}\right), \quad \tilde{K}=\left(\begin{array}{ccccc}
0 & 0 & 0 & 0 & 0 \\
0.4 & 0 & 0 & 0 & 0 \\
0.2 & 0 & 0 & 0 & 0 \\
0.45 & 0 & 0 & 0 & 0 \\
0.1 & 0 & 0 & 0 & 0
\end{array}\right)
$$

$\rho(\cdot)$ is the spectral radius of the corresponding iterative method. Numerical results for Example 1 are shown in Fig. 1. 


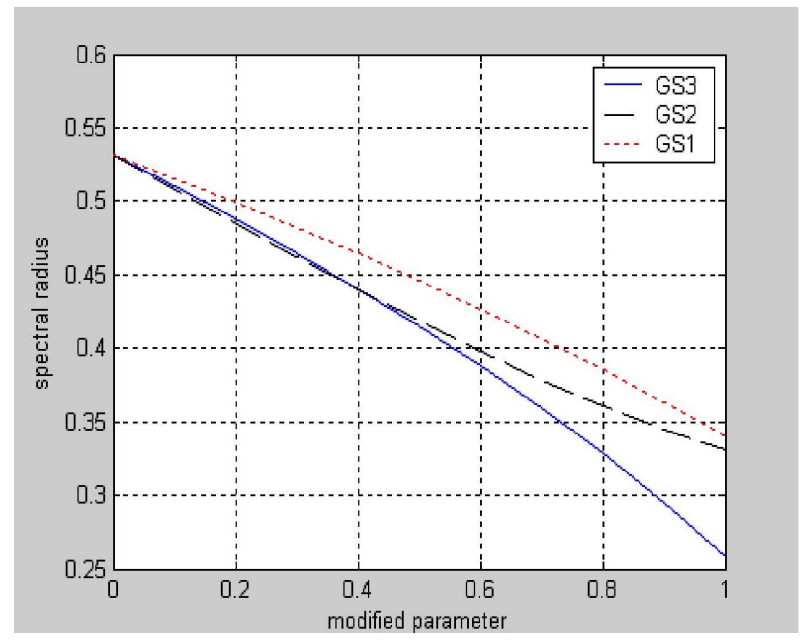

Fig. 1.

From the above numerical examples, we see that the preconditioning effectiveness of $(I+\alpha S+\beta \tilde{K})$-type preconditioners constructed in this paper is obvious.

\section{Conclusion}

In this paper, we have presented the new preconditioned Gauss-Seidel iterative method for the $Z$-matrices linear systems. It remains to construct the comparison theorems for the iterative methods. We conclude that the rate of convergence of the new method in this paper is faster than the rate of convergence of the methods in [5] and [7] by theoretical analysis and numerical examples.

\section{References}

[1] A. Berman and R. J. Plemmons, Nonnegative Matrices in the Mathematical Sciences, Society for Industrial and Applied Mathematics (SIAM), Philadelphia, PA, 1994.

[2] A. D. Gunawardena, S. K. Jain, and L. Snyder, Modified iterative methods for consistent linear systems, Linear Algebra Appl. 154/156 (1991), 123-143.

[3] A. Hadjidimos, D. Noutsos, and M. Tzoumas, More on modifications and improvements of classical iterative schemes for M-matrices, Linear Algebra Appl. 364 (2003), 253-279.

[4] J. Hu, The iterative methods of the linear equations, Beijing: Science Press (1997), 63-64.

[5] T. Kohno, H. Kotakemori, H. Niki, and M. Usui, Improving the modified Gauss-Seidel method for Z-matrices, Linear Algebra Appl. 267 (1997), 113-123.

[6] H. Kotakemori, H. Niki, and N. Okamoto, Accelerated iterative method for Z-matrices, J. Comput. Appl. Math. 75 (1996), no. 1, 87-97.

[7] J. Li and T. Huang, Preconditioning methods for Z-matrices, Acta Math. Sci. Ser. A Chin. Ed. 25 (2005), no. 1, 5-10.

[8] J. P. Milaszewicz, Improving Jacobi and Gauss-Seidel iterations, Linear Algebra Appl. 93 (1987), 161-170. 
[9] X. Shao, Z. Li, and C. Li, Modified SOR-like method for the augmented system, Int. J. Comput. Math. 84 (2007), no. 11, 1653-1662.

HAILONG SHEN

College of Sciences or School of Information Engineering

NORTHEASTERN University

Shenyang 110-004, P. R. China

E-mail address: hailong_shen@126.com

Xinhui ShaO

College of Sciences

NORTHEASTERN UNIVERSiTY

Shenyang 110-004, P. R. China

E-mail address: xinhui1002@126.com

ZhenXING HUANG

College of Sciences

Northeastern University

Shenyang 110-004, P. R. China

Chunji Li

Institute of System Science

College of Sciences

NORTHEASTERN UNIVERSITY

ShenYang 110-004, P. R. China

E-mail address: chunjili2000@yahoo.com.cn 